\title{
Aggregating preferences rankings with variable weights
}

\author{
Bonifacio Llamazares*, Teresa Peña \\ IMUVA (Institute of Mathematics) and Department of Applied Economics, University of Valladolid, Avda. Valle de Esgueva 6 , \\ 47011 Valladolid, Spain.
}

\begin{abstract}
One of the most important issues for aggregating preferences rankings is the determination of the weights associated with the different ranking places. To avoid the subjectivity in determining the weights, Cook and Kress (1990) suggested evaluating each candidate with the most favorable scoring vector for him/her. With this purpose, various models based on Data Envelopment Analysis have appeared in the literature. Although these methods do not require predetermine the weights subjectively, some of them have a serious drawback: the relative order between two candidates may be altered when the number of first, second, ..., $k$ th ranks obtained by other candidates changes, although there is not any variation in the number of first, second, $\ldots, k$ th ranks obtained by both candidates. In this paper we propose a model that allows each candidate to be evaluated with the most favorable weighting vector for him/her and avoids the previous drawback. Moreover, in some cases, we give a closed expression for the score assigned with our model to each candidate.
\end{abstract}

Keywords: Decision analysis, Scoring rules, Variable weights.

\section{Introduction}

This paper deals with voting systems in which each voter (of a collective of $n$ individuals) selects $k$ candidates from a set of $m$ candidates, $\left\{A_{1}, \ldots, A_{m}\right\}$, and ranks them from top to $k$ th place. In this context, scoring rules are well-known systems to get a social ranking or a winning candidate. These procedures are based on fixed scores, which are assigned to the different ranks obtained by the candidates. Thus, the score obtained by the candidate $A_{i}$ is $Z_{i}=\sum_{j=1}^{k} v_{i j} w_{j}$, where $v_{i j}$ is the number of $j$ th place ranks that candidate $A_{i}$ occupies and $\left(w_{1}, \ldots, w_{k}\right)$ is the scoring vector used. Once the scores are obtained for all candidates, they

\footnotetext{
${ }^{*}$ Corresponding author. Tel.: +34-983-186544; fax: +34-983-423299.

Email addresses: boni@eco.uva.es (Bonifacio Llamazares), maitepe@eco.uva.es (Teresa Peña)
} 
are ordered according to these scores. The plurality rule, where $w_{1}=1$ and $w_{j}=0$ for all $j \in\{2, \ldots, k\}$, and the Borda rule, where $k=m$ and $w_{j}=m-j$ for all $j \in\{1, \ldots, m\}$, are possibly the best known examples of scoring rules.

One of the most important issues in the field of scoring rules is the determination of the scoring vector to use, because a candidate that is not the winner with the scoring vector imposed initially could be it if another one is used. For instance, the Formula One World Championship uses $k=10$ and the scoring vector $(25,18,15,12,10,8,6,4,2,1)$ for obtaining the winner of the championship. In 2010, the winner was Sebastian Vettel, followed by Fernando Alonso. However, if the scoring vector utilized in the Motorcycle World Championship had been used, $\left(w_{1}, \ldots, w_{15}\right)=(25,20,16,13,11,10,9,8,7,6,5,4,3,2,1)$, then the winner would have been Fernando Alonso.

To avoid this problem, Cook \& Kress [5] suggested evaluating each candidate with the most favorable scoring vector for him/her. With this purpose, they introduced Data Envelopment Analysis (DEA) in this context. The model DEA/AR proposed by these authors is

$$
\begin{aligned}
Z_{o}^{*}(\varepsilon)=\max & \sum_{j=1}^{k} v_{o j} w_{j}, \\
\text { s.t. } \quad & \sum_{j=1}^{k} v_{i j} w_{j} \leq 1, \quad i=1, \ldots, m, \\
& w_{j}-w_{j+1} \geq d(j, \varepsilon), \quad j=1, \ldots, k-1, \\
& w_{k} \geq d(k, \varepsilon),
\end{aligned}
$$

where $\varepsilon \geq 0$ and the functions $d(j, \varepsilon)$, called the discrimination intensity functions, are nonnegative and nondecreasing in $\varepsilon$. Furthermore, $d(j, 0)=0$ for all $j \in\{1, \ldots, k\}$.

The principal drawback of this procedure is that several candidates are often efficient, i.e., they achieve the maximum attainable score $\left(Z_{o}^{*}=1\right)$. To avoid this weakness, Cook \& Kress [5] proposed to maximize the gap between consecutive weights of the scoring vector and, in this way, to reduce the feasible set of 
Problem (1). Thus, the model considered by these authors is

$$
\begin{array}{ll}
\max & \varepsilon, \\
\text { s.t. } & \sum_{j=1}^{k} v_{i j} w_{j} \leq 1, \quad i=1, \ldots, m, \\
& w_{j}-w_{j+1} \geq d(j, \varepsilon), \quad j=1, \ldots, k-1, \\
& w_{k} \geq d(k, \varepsilon),
\end{array}
$$

where $\varepsilon$ and the functions $d(j, \varepsilon)$ satisfy the conditions imposed in Model (1). Cook \& Kress [5] demonstrated that, at optimality, at least one of the constraints in (2a) and all the constraints in (2b) hold as equalities. The candidate(s) $A_{i}$ for which $\sum_{j=1}^{k} v_{i j} w_{j}=1$ are the winning candidates (see Cook \& Kress [5, p. 1308]). The candidate(s) in second place can be found by re-solving Model (2) after deleting the binding constraint(s) from (2a). This process can be repeated until the order of all candidates is fixed.

Since all the constraints in (2b) hold as equalities, Model (2) can be written as

$$
\begin{array}{ll}
\max & \varepsilon, \\
\text { s.t. } & \sum_{j=1}^{k}\left(\sum_{l=j}^{k} d(l, \varepsilon)\right) v_{i j} \leq 1, \quad i=1, \ldots, m, \\
& \varepsilon \geq 0 .
\end{array}
$$

Cook \& Kress [5] gave a closed form solution for this model when $d(j, \varepsilon)=g(j) h(\varepsilon)$ and $h(\varepsilon)$ is strictly monotonic increasing. Later, Green et al. [10] noticed that, in this case, the previous procedure amounts to the scoring rule given by the weights $w_{j}=\sum_{l=j}^{k} g(l)$. In the general case, this result is true when we only seek the winning candidates; that is, regardless of the discrimination intensity functions used, the winning candidates provided by Model (3) are the same as those obtained with a scoring rule: if $\varepsilon^{*}$ is the optimum value of Model (3), the winning candidates are those that, at optimality, satisfy

$$
\sum_{j=1}^{k}\left(\sum_{l=j}^{k} d\left(l, \varepsilon^{*}\right)\right) v_{i j}=1 .
$$

Therefore, they are the same candidates than those obtained with the scoring rules given by $w_{j}=$ $\sum_{l=j}^{k} d\left(l, \varepsilon^{*}\right)$. Consequently, when Model (3) is used, the aim pursued by Cook \& Kress [5] (evaluating each candidate with the most favorable scoring vector for him/her) is not reached.

Since the pioneering work of Cook \& Kress [5], several models have appeared in the literature in order to deal with this kind of problems (see Green et al. [10], Hashimoto [11], Noguchi et al. [16], Obata \& Ishii 
[17], Hashimoto \& Wu [12], Foroughi et al. [8], Foroughi \& Tamiz [9], Wang \& Chin [21], Wang et al. [22], Wang et al. [23], Wang et al. [20], Wu et al. [24], Amin \& Sadeghi [1], Soltanifar et al. [18], Contreras [4], Hosseinzadeh Lotfi \& Fallahnejad [13], Ebrahimnejad [6], Hosseinzadeh Lotfi et al. [14] and Foroughi \& Aouni [7]). A thorough analysis of some of them can be found in Llamazares \& Peña [15]. In this work the authors show that an important shortcoming of some of these models is that the relative order between two candidates may be altered when the number of first, second, $\ldots, k$ th ranks obtained by other candidates changes, although there is not any variation in the number of first, second, $\ldots, k$ th ranks obtained by both candidates. It is worth noting that this behavior is very questionable from the point of view of Social Choice Theory.

In this paper we propose a model that avoids the previous problem. To do this, from Cook and Kress's model, we put together in a single restriction the constraints of the candidates that are not being evaluated (Section 2). The main shortcoming of this initial model is that several candidates could be winners. In Section 3 we solve this problem by removing in the initial model the constraint of the evaluated candidate. On the other hand, in this aggregating preferences rankings' framework, the models that use non-null discrimination intensity functions have the following drawback: it is necessary to choose a specific value for $\varepsilon$ and the winner may change depending on the value of $\varepsilon$. To avoid this problem, in Section 4 we consider the average of the function that provides, for each value of $\varepsilon$, the score obtained for each candidate. Moreover, for some specific values of $d(j, \varepsilon)$, we give a closed expression for the scores assigned to the candidates. These expressions allow us to get the winning candidates without solving the proposed model. Finally, some concluding remarks are made in Section 5.

\section{An initial model}

The initial model that we propose is based on that of Cook \& Kress [5] but putting together in a single restriction the constraints of the candidates that are not being evaluated. So, we replace the constraints relative to the candidates that are not being evaluated,

$$
\sum_{j=1}^{k} w_{j} v_{i j} \leq 1 \quad i=1, \ldots, m, i \neq o,
$$

by their sum,

$$
\sum_{\substack{i=1 \\ i \neq o}}^{m} \sum_{j=1}^{k} w_{j} v_{i j} \leq m-1 .
$$


Since

$$
\sum_{\substack{i=1 \\ i \neq o}}^{m} \sum_{j=1}^{k} w_{j} v_{i j}=\sum_{j=1}^{k} w_{j} \sum_{\substack{i=1 \\ i \neq o}}^{m} v_{i j},
$$

and $v_{o j}$ is the number of $j$ th place ranks that candidate $A_{o}$ occupies, the number of $j$ th place ranks obtained by the remaining candidates is $n-v_{o j}$, where $n$ is the number of voters. Therefore, the sum constraint can be written as follows:

$$
\sum_{j=1}^{k} w_{j}\left(n-v_{o j}\right) \leq m-1,
$$

and the model proposed is

$$
\begin{aligned}
\Theta_{o}^{*}=\max & \sum_{j=1}^{k} v_{o j} w_{j}, \\
\text { s.t. } \quad & \sum_{j=1}^{k} v_{o j} w_{j} \leq 1, \\
& \sum_{j=1}^{k}\left(n-v_{o j}\right) w_{j} \leq m-1, \\
& w_{j}-w_{j+1} \geq d(j, \varepsilon), \quad j=1, \ldots, k-1, \\
& w_{k} \geq d(k, \varepsilon) .
\end{aligned}
$$

It is worth noting that the constraint

$$
\sum_{j=1}^{k}\left(n-v_{o j}\right) w_{j} \leq m-1
$$

(and then the feasible set of Model (4)) do not change when the number of ranks obtained by the candidates that are not being evaluated at that moment is altered. So, the score obtained by each candidate (and therefore the relative order between two candidates) do not depend on the individual evaluations of the remaining candidates.

Note that, for the sake of simplicity, we have used $\Theta_{o}^{*}$ instead of $\Theta_{o}^{*}(\varepsilon)$. Moreover, in order to facilitate the analysis of this model, in the following lemma we give an alternative representation of it. 
Lemma 1. Model (4) can be expressed as

$$
\begin{aligned}
\Theta_{o}^{*}=\max & \sum_{j=1}^{k} V_{o j} W_{j}+\sum_{j=1}^{k} V_{o j} d(j, \varepsilon), \\
\text { s.t. } \quad & \sum_{j=1}^{k} V_{o j} W_{j}+\sum_{j=1}^{k} V_{o j} d(j, \varepsilon) \leq 1, \\
& \sum_{j=1}^{k}\left(j n-V_{o j}\right) W_{j}+\sum_{j=1}^{k}\left(j n-V_{o j}\right) d(j, \varepsilon) \leq m-1, \\
& W_{j} \geq 0, \quad j=1, \ldots, k,
\end{aligned}
$$

where

$$
\begin{aligned}
W_{j} & =w_{j}-w_{j+1}-d(j, \varepsilon), \quad \text { for all } j \in\{1, \ldots, k-1\}, \\
W_{k} & =w_{k}-d(k, \varepsilon), \\
V_{o j} & =\sum_{l=1}^{j} v_{o l}, \quad \text { for all } j \in\{1, \ldots, k\} .
\end{aligned}
$$

It is worth noting that the values $V_{o j}=\sum_{l=1}^{j} v_{o l}$ are called cumulative standing (see, for instance, Green et al. [10] and Stein et al. [19]), and that satisfy $0 \leq V_{o 1} \leq \cdots \leq V_{o k} \leq n$.

Now, if we consider

$$
\begin{aligned}
& \delta_{o 1}=1-\sum_{j=1}^{k} V_{o j} d(j, \varepsilon), \\
& \delta_{o 2}=(m-1)-\sum_{j=1}^{k}\left(j n-V_{o j}\right) d(j, \varepsilon),
\end{aligned}
$$

the previous model can be written as

$$
\begin{aligned}
\Theta_{o}^{*}=\max & \sum_{j=1}^{k} V_{o j} W_{j}+\left(1-\delta_{o 1}\right), \\
\text { s.t. } \quad & \sum_{j=1}^{k} V_{o j} W_{j} \leq \delta_{o 1}, \\
& \sum_{j=1}^{k}\left(j n-V_{o j}\right) W_{j} \leq \delta_{o 2}, \\
& W_{j} \geq 0, \quad j=1, \ldots, k .
\end{aligned}
$$

In order to guarantee that the above problem is feasible, we need to impose the conditions $\delta_{o 1} \geq 0$ and $\delta_{o 2} \geq 0$. Moreover, it is worth noting that the feasible set of the previous model varies according 
to the evaluated candidate. Therefore, if we want that all the feasible sets are non-empty, we need that $\min _{o=1, \ldots, m} \delta_{o 1} \geq 0$ and $\min _{o=1, \ldots, m} \delta_{o 2} \geq 0$.

In the following theorem we give the optimal value for Model (5).

Theorem 1. Consider Model (5). The following statements hold:

1. If $V_{o 1}=n$, then $\Theta_{o}^{*}=1$.

2. If $V_{o 1}<n$, then

$$
\Theta_{o}^{*}= \begin{cases}1-\left(\delta_{o 1}-\delta_{o 2} V_{o}^{*}\right) & \text { if } \delta_{o 2}=0 \text { or } \delta_{o 1} / \delta_{o 2}>V_{o}^{*}, \\ 1 & \text { if } \delta_{o 1} / \delta_{o 2} \leq V_{o}^{*},\end{cases}
$$

where $V_{o}^{*}=\max _{j=1, \ldots, k} \frac{V_{o j}}{j n-V_{o j}}$.

The main shortcoming of this model is that usually provides more of one winning candidate. For instance, consider $d(j, \varepsilon)=0$ for all $j \in\{1, \ldots, k\}$. Then $\delta_{o 1}=1$ and $\delta_{o 2}=m-1$. According to Theorem 1 , the winning candidates are those that $V_{o 1}=n$ or $V_{o}^{*} \geq 1 /(m-1)$. This fact may produce unfair outcomes for some candidates. For instance, consider $k \geq 2$ and two or more candidates where one of them, A, obtains all the first ranks and another, $\mathrm{B}$, gets all the second ranks. Obviously $\Theta_{\mathrm{A}}^{*}=1$ and, given that $V_{\mathrm{B}}^{*}=1 \geq 1 /(m-1)$, we also have $\Theta_{\mathrm{B}}^{*}=1$. Therefore, both are winning candidates although it seems logical to think that candidate A should be the winner.

Multiple winning candidates also appear when we consider non-null discrimination intensity functions. For instance, consider Table 1, taken from Cook \& Kress [5, p. 1309], and the following discrimination intensity functions (also considered by them): $d(j, \varepsilon)=\varepsilon, d(j, \varepsilon)=\varepsilon / j$ and $d(j, \varepsilon)=\varepsilon / j$ !

Table 1: Ranks obtained by each candidate.

\begin{tabular}{ccccc}
\hline Candidate & $v_{i 1}$ & $v_{i 2}$ & $v_{i 3}$ & $v_{i 4}$ \\
\hline $\mathrm{A}$ & 3 & 3 & 4 & 3 \\
$\mathrm{~B}$ & 4 & 5 & 5 & 2 \\
$\mathrm{C}$ & 6 & 2 & 3 & 2 \\
$\mathrm{D}$ & 6 & 2 & 2 & 6 \\
$\mathrm{E}$ & 0 & 4 & 3 & 4 \\
$\mathrm{~F}$ & 1 & 4 & 3 & 3 \\
\hline
\end{tabular}


For each candidate, we show in Table 2 the value $V_{o}^{*}$, and also the values $\delta_{o 1} / \delta_{o 2}$ for each family of discrimination intensity functions considering two values of $\varepsilon$. One of them is the maximum value that we can take in order to both $\min _{o=1, \ldots, m} \delta_{o 1}$ and $\min _{o=1, \ldots, m} \delta_{o 2}$ be greater than or equal to zero; and the other is a value close to the half of the previous one.

Table 2: Values of $V_{o}^{*}$ and $\delta_{o 1} / \delta_{o 2}$ for the candidates of Table 1 .

\begin{tabular}{cccccccc} 
& & \multicolumn{2}{c}{$d(j, \varepsilon)=\varepsilon$} & \multicolumn{2}{c}{$d(j, \varepsilon)=\varepsilon / j$} & \multicolumn{2}{c}{$d(j, \varepsilon)=\varepsilon / j !$} \\
\hline Candidate & $V_{o}^{*}$ & $\varepsilon=0.01$ & $\varepsilon=1 / 43$ & $\varepsilon=0.03$ & $\varepsilon=3 / 52$ & $\varepsilon=0.04$ & $\varepsilon=8 / 99$ \\
\hline A & 0.2000 & 0.2048 & 0.2340 & 0.2091 & 0.2468 & 0.2102 & 0.2488 \\
B & 0.3043 & 0.1662 & 0.0000 & 0.1557 & 0.0070 & 0.1623 & 0.0437 \\
C & 0.4286 & 0.1834 & 0.0943 & 0.1585 & 0.0177 & 0.1502 & 0.0000 \\
D & 0.4286 & 0.1765 & 0.0545 & 0.1538 & 0.0000 & 0.1508 & 0.0020 \\
E & 0.1594 & 0.2422 & 0.5676 & 0.2800 & 0.7455 & 0.2839 & 0.7192 \\
F & 0.1594 & 0.2308 & 0.4500 & 0.2554 & 0.5401 & 0.2561 & 0.5120 \\
\hline
\end{tabular}

When $d(j, \varepsilon)=0$ for all $j \in\{1, \ldots, 4\}, \delta_{o 1} / \delta_{o 2}=0.2$ for all candidates. Therefore, the winning candidates are A, B, C and D. When the discrimination intensity functions are not null, B, C and D continue to be winning candidates in all considered cases. This shortcoming of multiple winning candidates is solved in the next section.

\section{Breaking ties}

As we have seen in the previous section, it is usual that several candidates achieve the maximum score when Model (5) is applied. One possibility to avoid this fact is to remove the constraint relative to the evaluated candidate. This procedure is the same as that used in the DEA exclusion method (see Andersen $\&$ Petersen [2] and, in this context, Hashimoto [11]). Therefore, the model proposed is 


$$
\begin{aligned}
\Lambda_{o}^{*}=\max & \sum_{j=1}^{k} v_{o j} w_{j}, \\
\text { s.t. } & \sum_{j=1}^{k}\left(n-v_{o j}\right) w_{j} \leq m-1, \\
& w_{j}-w_{j+1} \geq d(j, \varepsilon), \quad j=1, \ldots, k-1, \\
& w_{k} \geq d(k, \varepsilon) .
\end{aligned}
$$

If, as in the previous section, we consider $W_{j}=w_{j}-w_{j+1}-d(j, \varepsilon)$ for all $j \in\{1, \ldots, k-1\}, W_{k}=$ $w_{k}-d(k, \varepsilon)$ and $V_{o j}=\sum_{l=1}^{j} v_{o l}$ for all $j \in\{1, \ldots, k\}$, then Model (6) becomes

$$
\begin{aligned}
\Lambda_{o}^{*}=\max & \sum_{j=1}^{k} V_{o j} W_{j}+\sum_{j=1}^{k} V_{o j} d(j, \varepsilon), \\
\text { s.t. } & \sum_{j=1}^{k}\left(j n-V_{o j}\right) W_{j}+\sum_{j=1}^{k}\left(j n-V_{o j}\right) d(j, \varepsilon) \leq m-1, \\
& W_{j} \geq 0, \quad j=1, \ldots, k .
\end{aligned}
$$

Moreover, if we put

$$
\begin{aligned}
& \delta_{o}=\sum_{j=1}^{k} V_{o j} d(j, \varepsilon), \\
& \delta_{o 2}=(m-1)-\sum_{j=1}^{k}\left(j n-V_{o j}\right) d(j, \varepsilon),
\end{aligned}
$$

the previous model can be written as

$$
\begin{aligned}
\Lambda_{o}^{*}=\max & \sum_{j=1}^{k} V_{o j} W_{j}+\delta_{o}, \\
\text { s.t. } & \sum_{j=1}^{k}\left(j n-V_{o j}\right) W_{j} \leq \delta_{o 2}, \\
& W_{j} \geq 0, \quad j=1, \ldots, k .
\end{aligned}
$$

The condition $\delta_{o 2} \geq 0$ is necessary in order to ensure that the above problem is feasible. As in Model (5), the feasible set varies according to the evaluated candidate. So, in order to all the feasible sets are non-empty we need that $\min _{o=1, \ldots, m} \delta_{o 2} \geq 0$. 
When $V_{o 1}=n$, then $V_{o 2}=\cdots=V_{o k}=n$ and the feasible set is

$$
S=\left\{\left(W_{1}, \ldots, W_{k}\right) \in \mathbb{R}_{+}^{k} \mid n W_{2}+2 n W_{3}+\cdots+(k-1) n W_{k} \leq \delta_{o 2}\right\}
$$

Therefore Model (7) is unbounded and, consequently, candidate $A_{o}$ is the winner. In the following theorem we give the optimal value of this program for the remaining cases.

Theorem 2. Consider Model (7) when $V_{o 1}<n$. Then $\Lambda_{o}^{*}=\delta_{o 2} V_{o}^{*}+\delta_{o}$, where $V_{o}^{*}=\max _{j=1, \ldots, k} \frac{V_{o j}}{j n-V_{o j}}$.

It is worth noting that the value of $\Lambda_{o}^{*}$ coincides with that of $\Theta_{o}^{*}$ when $\delta_{o 2}=0$ or $\delta_{o 1} / \delta_{o 2}>V_{o}^{*}$. So, the value $\delta_{o 2} V_{o}^{*}+\delta_{o}$ can be seen as a natural way to break ties in Model (5).

In the following subsections we analyze the proposed model according to whether the discrimination intensity functions are null or not.

\subsection{Null discrimination intensity functions}

Consider $d(j, \varepsilon)=0$ for all $j \in\{1, \ldots, k\}$. In this case $\delta_{o}=0, \delta_{o 2}=m-1$ and Model (7) becomes

$$
\begin{aligned}
\Lambda_{o}^{*}=\max & \sum_{j=1}^{k} V_{o j} W_{j}, \\
\text { s.t. } & \sum_{j=1}^{k}\left(j n-V_{o j}\right) W_{j} \leq m-1, \\
& W_{j} \geq 0, \quad j=1, \ldots, k .
\end{aligned}
$$

By Theorem 2, if $V_{o 1}<n$, the score obtained by the candidate $A_{o}$ is

$$
\Lambda_{o}^{*}=(m-1) \max _{j=1, \ldots, k} \frac{V_{o j}}{j n-V_{o j}} .
$$

Let $A_{o}$ and $A_{i}$ be two candidates such that $V_{o 1}<n$ and $V_{i 1}<n$. Given that

$$
\frac{V_{o j}}{j n-V_{o j}}>\frac{V_{i l}}{l n-V_{i l}} \Leftrightarrow V_{o j}\left(\ln -V_{i l}\right)>V_{i l}\left(j n-V_{o j}\right) \Leftrightarrow V_{o j} l>V_{i l j} \quad \Leftrightarrow \frac{V_{o j}}{j}>\frac{V_{i l}}{l}
$$

for all $j, l \in\{1, \ldots, k\}$, we have

$$
\Lambda_{o}^{*}>\Lambda_{i}^{*} \Leftrightarrow \max _{j=1, \ldots, k} \frac{V_{o j}}{j}>\max _{j=1, \ldots, k} \frac{V_{i j}}{j}
$$

On the other hand, if $A_{o}$ is a candidate such that $V_{o 1}=n$, then $n=\max _{j=1, \ldots, k} \frac{V_{o j}}{j}>\max _{j=1, \ldots, k} \frac{V_{i j}}{j}$ for all $i \neq o$; that is, $A_{o}$ is also the winner when we consider the score $\max _{j=1, \ldots, k} \frac{V_{o j}}{j}$. Taking into account the previous 
remarks, the rank given by Model (8) is the same as obtained using the expression $\widehat{Z}_{o}=\max _{j=1, \ldots, k} \frac{V_{o j}}{j}$, which is simpler. This value, $\widehat{Z}_{o}$, is used to determine the winning candidate when we take the $L_{1}$-norm and $d(j, \varepsilon)=0$ in the model given by Obata \& Ishii [17] (see Llamazares \& Peña [15, p. 720]).

It is worth noting that the winning candidate obtained with this model may be, in some cases, less controversial than that obtained with other procedures. For instance, consider Table 3, where it is shown the number of first, second, third and fourth ranks obtained by four candidates.

Table 3: Ranks obtained by each candidate.

\begin{tabular}{ccccc}
\hline Candidate & $v_{i 1}$ & $v_{i 2}$ & $v_{i 3}$ & $v_{i 4}$ \\
\hline $\mathrm{A}$ & 5 & 0 & 2 & 2 \\
$\mathrm{~B}$ & 4 & 0 & 5 & 0 \\
$\mathrm{C}$ & 0 & 0 & 2 & 7 \\
$\mathrm{D}$ & 0 & 9 & 0 & 0 \\
\hline
\end{tabular}

If we apply the Borda rule, which is superior in many respects to other scoring rules (see, for instance, Brams \& Fishburn [3, p. 216]), the following scores are obtained:

$$
Z_{\mathrm{A}}=17, \quad Z_{\mathrm{B}}=17, \quad Z_{\mathrm{C}}=2, \quad Z_{\mathrm{D}}=18 .
$$

Therefore, candidate D is the winner despite not getting any first rank. However, when Model (8) is applied for each of the four candidates, and taking into account the equivalence (9), the following rank is obtained:

$$
\mathrm{A}>\mathrm{D}>\mathrm{B}>\mathrm{C} .
$$

Note that candidate A is the Condorcet winner (that is, a candidate that pairwise defeats every other candidate) since he/she has obtained more than half of the first ranks. As we show in the following proposition, Model (8) guarantees this property: if a candidate receives more than half of the first ranks, then he/she is the winner.

Proposition 1. Let $A_{o}$ be a candidate such that $v_{o 1}>n / 2$. Then $A_{o}>A_{i}$ for all $i \neq o$.

\subsection{Non-null discrimination intensity functions}

When the discrimination intensity functions are not null we have to fix the value of $\varepsilon$, as well as choose these functions. Moreover, as we shall see below, the choice of this value may determine the winning 
candidate.

Note that, although to simplify the notation we have used $\Lambda_{o}^{*}, \delta_{o}$ and $\delta_{o 2}$, these values are actually functions of $\varepsilon, \Lambda_{o}^{*}(\varepsilon), \delta_{o}(\varepsilon)$ and $\delta_{o 2}(\varepsilon)$. Furthermore, since the functions $d(j, \varepsilon)$ are nondecreasing in $\varepsilon$, the feasible set of Model (6) (or, equivalently, the feasible set of Model (7)) does not increase when the value of $\varepsilon$ increases. Therefore $\Lambda_{o}^{*}(\varepsilon)$ is a nonincreasing function; that is, if $\varepsilon_{1}>\varepsilon_{2}$, then $\Lambda_{o}^{*}\left(\varepsilon_{1}\right) \leq \Lambda_{o}^{*}\left(\varepsilon_{2}\right)$.

Consider, for instance, $d(j, \varepsilon)=\varepsilon$ for all $j \in\{1, \ldots, k\}$. Then

$$
\delta_{o}(\varepsilon)=\varepsilon \sum_{j=1}^{k} V_{o j} \quad \text { and } \quad \delta_{o 2}(\varepsilon)=(m-1)+\varepsilon\left(\sum_{j=1}^{k} V_{o j}-\frac{n k(k+1)}{2}\right) .
$$

According to Theorem 2, we have

$$
\Lambda_{o}^{*}(\varepsilon)=(m-1) V_{o}^{*}+\varepsilon\left(\left(V_{o}^{*}+1\right) \sum_{j=1}^{k} V_{o j}-V_{o}^{*} \frac{n k(k+1)}{2}\right),
$$

that is, the graph of $\Lambda_{o}^{*}(\varepsilon)$ is a straight line. Moreover, since $\Lambda_{o}^{*}(\varepsilon)$ is a nonincreasing function, the slope of this straight line is negative or null.

Consider now the example given by Table 1 . In this case

$$
\Lambda_{o}^{*}(\varepsilon)=5 V_{o}^{*}+\varepsilon\left(\left(V_{o}^{*}+1\right) \sum_{j=1}^{4} V_{o j}-200 V_{o}^{*}\right) .
$$

When we focus on candidates B and D we have

$$
\Lambda_{\mathrm{B}}^{*}(\varepsilon)=\frac{35}{23}-\frac{110}{23} \varepsilon, \quad \Lambda_{\mathrm{D}}^{*}(\varepsilon)=\frac{15}{7}-\frac{200}{7} \varepsilon .
$$

Both functions appear drawn in Figure 1 (Note that we have considered different scales on both axes; a hundredth on the $x$-axis is equal to one unit on the $y$-axis).

As we can see in Figure 1, when we take values of $\varepsilon$ less than 10/383 we have D $>$ B. However, if the values of $\varepsilon$ are greater than 10/383, then B $>$ D. In Table 4 we show this behavior for two specific values of $\varepsilon, \varepsilon=0.01$ and $\varepsilon=5 / 178$. This last value is the maximum possible value for $\varepsilon$, that is, the maximum value for which $\min _{o=1, \ldots, m} \delta_{o 2}(\varepsilon) \geq 0$.

Therefore, it is not obvious how to choose a specific value of $\varepsilon$. One possibility would be to always take the maximum possible value for $\varepsilon$ (in the same spirit that in Cook and Kress' model), although in the previous example (see Figure 1), it does seem the best choice. On the other hand, this solution has a serious shortcoming: the order between two candidates may depend on the ranks obtained by other candidates. For instance, suppose that candidates A, E and F obtain the ranks shown in Table 5, which are different from 


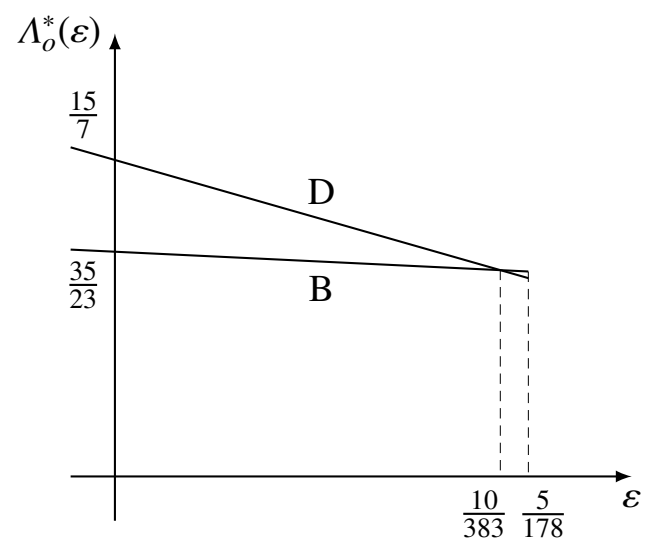

Figure 1: Graphs of the functions $\Lambda_{\mathrm{B}}^{*}(\varepsilon)$ and $\Lambda_{\mathrm{D}}^{*}(\varepsilon)$.

Table 4: Values of $\delta_{o 2}(\varepsilon)$ and $\Lambda_{o}^{*}(\varepsilon)$ for the candidates of Table 1 .

\begin{tabular}{ccccc}
\cline { 2 - 5 } & \multicolumn{2}{c}{$\varepsilon=0.01$} & \multicolumn{2}{c}{$\varepsilon=5 / 178$} \\
\hline Candidate & $\delta_{o 2}(\varepsilon)$ & $\Lambda_{o}^{*}(\varepsilon)$ & $\delta_{o 2}(\varepsilon)$ & $\Lambda_{o}^{*}(\varepsilon)$ \\
\hline $\mathrm{A}$ & 3.32 & 0.9840 & 0.2809 & 0.9551 \\
$\mathrm{~B}$ & 3.43 & 1.4739 & 0.5899 & 1.3874 \\
$\mathrm{C}$ & 3.38 & 1.8286 & 0.4494 & 1.2600 \\
$\mathrm{D}$ & 3.40 & 1.8571 & 0.5056 & 1.3403 \\
$\mathrm{E}$ & 3.22 & 0.7333 & 0.0000 & 0.6180 \\
$\mathrm{~F}$ & 3.25 & 0.7681 & 0.0843 & 0.7157 \\
\hline
\end{tabular}

those shown in Table 1. Now, the maximum possible value for $\varepsilon$ is $5 / 194$, and, with this value, $\mathrm{D}>\mathrm{B}$ $\left(\Lambda_{\mathrm{B}}^{*}(\varepsilon)\right.$ and $\Lambda_{\mathrm{D}}^{*}(\varepsilon)$ are still the functions of Figure 1 and $5 / 194<10 / 383$; see also Table 5$)$.

A solution to avoid taking a fixed value of $\varepsilon$ is to consider the average of the functions $\Lambda_{o}^{*}(\varepsilon)$. Moreover, for each candidate $A_{o}$ we only consider the constraint $\delta_{o 2}(\varepsilon) \geq 0$ instead of the restriction $\min _{o=1, \ldots, m} \delta_{o 2}(\varepsilon) \geq 0$. This prevents that the average of the function $\Lambda_{o}^{*}(\varepsilon)$ may depend on the results obtained for the remaining candidates. This proposal will be developed in the next section. 
Table 5: New ranks and values of $\delta_{o 2}(\varepsilon)$ and $\Lambda_{o}^{*}(\varepsilon)$ for $\varepsilon=5 / 194$.

\begin{tabular}{ccccccc}
\hline Candidate & $v_{i 1}$ & $v_{i 2}$ & $v_{i 3}$ & $v_{i 4}$ & $\delta_{o 2}$ & $\Lambda_{o}^{*}(\varepsilon)$ \\
\hline $\mathrm{A}$ & 3 & 3 & 6 & 3 & 0.7732 & 1.1211 \\
$\mathrm{~B}$ & 4 & 5 & 5 & 2 & 0.9536 & 1.3985 \\
$\mathrm{C}$ & 6 & 2 & 3 & 2 & 0.8247 & 1.3328 \\
$\mathrm{D}$ & 6 & 2 & 2 & 6 & 0.8763 & 1.4065 \\
$\mathrm{E}$ & 0 & 0 & 1 & 4 & 0.0000 & 0.1546 \\
$\mathrm{~F}$ & 1 & 8 & 3 & 3 & 0.7990 & 1.1856 \\
\hline
\end{tabular}

\section{Averaging the functions $\Lambda_{o}^{*}(\varepsilon)$}

Consider again Model (7):

$$
\begin{aligned}
\Lambda_{o}^{*}(\varepsilon)=\max & \sum_{j=1}^{k} V_{o j} W_{j}+\delta_{o}(\varepsilon), \\
\text { s.t. } & \sum_{j=1}^{k}\left(j n-V_{o j}\right) W_{j} \leq \delta_{o 2}(\varepsilon), \\
& W_{j} \geq 0, \quad j=1, \ldots, k,
\end{aligned}
$$

where

$$
\begin{aligned}
& \delta_{o}(\varepsilon)=\sum_{j=1}^{k} V_{o j} d(j, \varepsilon), \\
& \delta_{o 2}(\varepsilon)=(m-1)-\sum_{j=1}^{k}\left(j n-V_{o j}\right) d(j, \varepsilon) .
\end{aligned}
$$

We have seen that when $V_{o 1}=n$, then the candidate $A_{o}$ is the winner. On the other hand, if $V_{o j}=0$ for all $j \in\{1, \ldots, k\}$, then $\Lambda_{o}^{*}(\varepsilon)=0$ for all $\varepsilon \geq 0$.

Consider now the remaining cases. When $d(j, \varepsilon)=0$ for all $j \in\{1, \ldots, k\}$, we know, from Subsection 3.1, that

$$
\Lambda_{o}^{*}(\varepsilon)=(m-1) \max _{j=1, \ldots, k} \frac{V_{o j}}{j n-V_{o j}},
$$

that is, $\Lambda_{o}^{*}(\varepsilon)$ does not depend on $\varepsilon$. If $d(j, \varepsilon)$ is not null for some $j \in\{1, \ldots, k\}$, Model (10) is feasible if and only if $\delta_{o 2}(\varepsilon) \geq 0$. Since $\delta_{o 2}(\varepsilon)$ is a nonincreasing function (because $d(j, \varepsilon)$ are nondecreasing in $\varepsilon$ ), we 
can consider the maximum value for which the feasible set is not-empty, that is,

$$
\varepsilon_{o}^{*}=\sup \left\{\varepsilon \geq 0 \mid \delta_{o 2}(\varepsilon) \geq 0\right\} .
$$

Once known the value of $\varepsilon_{o}^{*}$, the score assigned to the candidate $A_{o}$ is

$$
\widehat{\Lambda}_{o}=\frac{1}{\varepsilon_{o}^{*}} \int_{0}^{\varepsilon_{o}^{*}} \Lambda_{o}^{*}(\varepsilon) d \varepsilon
$$

that is, the average of the function $\Lambda_{o}^{*}(\varepsilon)$ (on the assumption that this value exists). Obviously, if $\varepsilon_{o}^{*}=0$, then we consider $\widehat{\Lambda}_{o}=\Lambda_{o}^{*}(0)$. Moreover, note that the value of $\varepsilon_{o}^{*}$ may not be finite. In this case, the value of $\widehat{\Lambda}_{o}$ could be defined as

$$
\widehat{\Lambda}_{o}=\lim _{b \rightarrow \infty} \frac{1}{b} \int_{0}^{b} \Lambda_{o}^{*}(\varepsilon) d \varepsilon
$$

In any case, from now on we suppose that $\varepsilon_{o}^{*}$ is finite, non-null and that $\int_{0}^{\varepsilon_{o}^{*}} \Lambda_{o}^{*}(\varepsilon) d \varepsilon$ exists. Given that

$$
\begin{aligned}
\Lambda_{o}^{*}(\varepsilon) & =\delta_{o 2}(\varepsilon) V_{o}^{*}+\delta_{o}(\varepsilon)=\left((m-1)-\sum_{j=1}^{k}\left(j n-V_{o j}\right) d(j, \varepsilon)\right) V_{o}^{*}+\sum_{j=1}^{k} V_{o j} d(j, \varepsilon) \\
& =(m-1) V_{o}^{*}+\sum_{j=1}^{k}\left(V_{o j}-\left(j n-V_{o j}\right) V_{o}^{*}\right) d(j, \varepsilon),
\end{aligned}
$$

we have

$$
\widehat{\Lambda}_{o}=(m-1) V_{o}^{*}+\frac{1}{\varepsilon_{o}^{*}} \int_{0}^{\varepsilon_{o}^{*}}\left(\sum_{j=1}^{k}\left(V_{o j}-\left(j n-V_{o j}\right) V_{o}^{*}\right) d(j, \varepsilon)\right) d \varepsilon .
$$

Now we focus on some specific cases of $d(j, \varepsilon)$. Consider $d(j, \varepsilon)=g(j) h(\varepsilon)$, where $h(\varepsilon)$ is strictly monotonic increasing in $\varepsilon$ (see Cook \& Kress [5, p. 1309]). In this case,

$$
\widehat{\Lambda}_{o}=(m-1) V_{o}^{*}+\frac{1}{\varepsilon_{o}^{*}}\left(\sum_{j=1}^{k}\left(V_{o j}-\left(j n-V_{o j}\right) V_{o}^{*}\right) g(j)\right) \int_{0}^{\varepsilon_{o}^{*}} h(\varepsilon) d \varepsilon
$$

In the following proposition we show the values of $\varepsilon_{o}^{*}$ and $\widehat{\Lambda}_{o}$ when $h(\varepsilon)=\varepsilon$, that is, $d(j, \varepsilon)=g(j) \varepsilon$.

Proposition 2. Consider Model (10) and $\widehat{\Lambda}_{o}$ given by $(11)$. If $d(j, \varepsilon)=g(j) \varepsilon$, then

$$
\varepsilon_{o}^{*}=\frac{m-1}{\sum_{j=1}^{k}\left(j n-V_{o j}\right) g(j)}
$$


and

$$
\widehat{\Lambda}_{o}=\frac{m-1}{2}\left(V_{o}^{*}+\left(\frac{n \sum_{j=1}^{k} j g(j)}{\sum_{j=1}^{k} V_{o j} g(j)}-1\right)^{-1}\right) .
$$

It is worth noting that the order among the candidates does not depend on the number of candidates; that is, in order to rank the candidates we can use the value $\widehat{\Lambda}_{o}$ or the following one:

$$
V_{o}^{*}+\left(\frac{n \sum_{j=1}^{k} j g(j)}{\sum_{j=1}^{k} V_{o j} g(j)}-1\right)^{-1} .
$$

We now show the values of $\widehat{\Lambda}_{o}$ for three specific cases proposed by Cook \& Kress [5]:

1. If $g(j)=1$, that is, $d(j, \varepsilon)=\varepsilon$, then

$$
\widehat{\Lambda}_{o}=\frac{m-1}{2}\left(V_{o}^{*}+\left(\frac{n k(k+1)}{2 \sum_{j=1}^{k} V_{o j}}-1\right)^{-1}\right) .
$$

2. If $g(j)=1 / j$, that is, $d(j, \varepsilon)=\varepsilon / j$, then

$$
\widehat{\Lambda}_{o}=\frac{m-1}{2}\left(V_{o}^{*}+\left(\frac{n k}{\sum_{j=1}^{k} \frac{V_{o j}}{j}}-1\right)^{-1}\right) .
$$

3. If $g(j)=1 / j$ !, that is, $d(j, \varepsilon)=\varepsilon / j$ !, then

$$
\widehat{\Lambda}_{o}=\frac{m-1}{2}\left(V_{o}^{*}+\left(\frac{n \sum_{j=1}^{k} \frac{1}{(j-1) !}}{\sum_{j=1}^{k} \frac{V_{o j}}{j !}}-1\right)^{-1}\right)
$$

Consider again the candidates of Table 1. In Table 6 we show the values of $\widehat{\Lambda}_{o}$ for the previous discrimination intensity functions. 
Table 6: Values of $\widehat{\Lambda}_{o}$ for the candidates of Table 1 .

\begin{tabular}{cccc}
\hline Candidate & $d(j, \varepsilon)=\varepsilon$ & $d(j, \varepsilon)=\varepsilon / j$ & $d(j, \varepsilon)=\varepsilon / j !$ \\
\hline $\mathrm{A}$ & 0.9762 & 0.9666 & 0.9548 \\
$\mathrm{~B}$ & 1.4456 & 1.4439 & 1.4481 \\
$\mathrm{C}$ & 1.6578 & 1.7418 & 1.8268 \\
$\mathrm{D}$ & 1.6964 & 1.7629 & 1.8235 \\
$\mathrm{E}$ & 0.7075 & 0.6414 & 0.5809 \\
$\mathrm{~F}$ & 0.7557 & 0.7122 & 0.6739 \\
\hline
\end{tabular}

As we can see, D is the winning candidate when $d(j, \varepsilon)=\varepsilon$ or $d(j, \varepsilon)=\varepsilon / j$, while $\mathrm{C}$ is the winner when $d(j, \varepsilon)=\varepsilon / j$ ! These results coincides with that obtained by Cook \& Kress [5] when $d(j, \varepsilon)=\varepsilon / j$ or $d(j, \varepsilon)=\varepsilon / j$ ! However, when $d(j, \varepsilon)=\varepsilon$, we get that $\mathrm{D}$ is the winner while the winning candidate with their model is B. In our case, as we can see from Figure 1, it seems reasonable that $\mathrm{D}$ be the winner. Note that in Figure 1 the functions $\Lambda_{\mathrm{B}}^{*}(\varepsilon)$ and $\Lambda_{\mathrm{D}}^{*}(\varepsilon)$ are plotted up to the value of $\varepsilon=5 / 178$. However, the averages of the functions $\Lambda_{\mathrm{B}}^{*}(\varepsilon)$ and $\Lambda_{\mathrm{D}}^{*}(\varepsilon)$ are calculated in the intervals [0,5/157] and [0,5/160], respectively.

\section{Concluding remarks}

Scoring rules are decision rules usually used in some areas (for instance, in sports competitions) to rank a set of candidates. The main problem that they present is the choice of the weights associated with the different ranking places. For this reason, several models have appeared in the literature trying to evaluate each candidate with the most favorable scoring vector for him/her. However, most of them are not fully convincing from the point of view of the Social Choice Theory. The score that our model assigns to each candidate (the average of the function provided for Model (10)) avoids the problems that the other models have. In addition to this, it is worth noting that, in some important cases, we give a closed expression for obtaining the scores of the candidates. So, it is not necessary to solve the proposed model.

\section{Acknowledgements}

This work is partially supported by the Spanish Ministry of Science and Innovation (Projects ECO200907332 and ECO2011-24200), and ERDF. 


\section{Appendix A. Proofs}

Proof of Lemma 1. Given Model (4), consider the following change of variables:

$$
\left\{\begin{array}{l}
W_{j}=w_{j}-w_{j+1}-d(j, \varepsilon), \quad \text { for all } j \in\{1, \ldots, k-1\}, \\
W_{k}=w_{k}-d(k, \varepsilon) .
\end{array}\right.
$$

In this case, $w_{j}=\sum_{l=j}^{k}\left(W_{l}+d(l, \varepsilon)\right)$ for all $j \in\{1, \ldots, k\}$ and

$$
\begin{aligned}
\sum_{j=1}^{k} v_{o j} w_{j} & =\sum_{j=1}^{k} v_{o j}\left(\sum_{l=j}^{k}\left(W_{l}+d(l, \varepsilon)\right)=\sum_{\substack{1 \leq j \leq k \\
j \leq l \leq k}} v_{o j}\left(W_{l}+d(l, \varepsilon)\right)=\sum_{1 \leq j \leq l \leq k} v_{o j}\left(W_{l}+d(l, \varepsilon)\right)\right. \\
& =\sum_{\substack{1 \leq l \leq k \\
1 \leq j \leq l}} v_{o j}\left(W_{l}+d(l, \varepsilon)\right)=\sum_{l=1}^{k}\left(W_{l}+d(l, \varepsilon)\right)\left(\sum_{j=1}^{l} v_{o j}\right)=\sum_{j=1}^{k}\left(W_{j}+d(j, \varepsilon)\right)\left(\sum_{l=1}^{j} v_{o l}\right),
\end{aligned}
$$

where the last equality is obtained by changing the role of $j$ and $l$.

If we denote $\sum_{l=1}^{j} v_{o l}$ by $V_{o j}$, then we have

$$
\sum_{j=1}^{k} v_{o j} w_{j}=\sum_{j=1}^{k} V_{o j} W_{j}+\sum_{j=1}^{k} V_{o j} d(j, \varepsilon) .
$$

Analogously,

$$
\begin{aligned}
\sum_{j=1}^{k}\left(n-v_{o j}\right) w_{j} & =\sum_{j=1}^{k}\left(n-v_{o j}\right)\left(\sum_{l=j}^{k}\left(W_{l}+d(l, \varepsilon)\right)=\sum_{\substack{1 \leq j \leq k \\
j \leq l \leq k}}\left(n-v_{o j}\right)\left(W_{l}+d(l, \varepsilon)\right)\right. \\
& =\sum_{1 \leq j \leq l \leq k}\left(n-v_{o j}\right)\left(W_{l}+d(l, \varepsilon)\right)=\sum_{\substack{1 \leq l \leq k \\
1 \leq j \leq l}}\left(n-v_{o j}\right)\left(W_{l}+d(l, \varepsilon)\right) \\
& =\sum_{l=1}^{k}\left(W_{l}+d(l, \varepsilon)\right)\left(\sum_{j=1}^{l}\left(n-v_{o j}\right)\right)=\sum_{l=1}^{k}\left(W_{l}+d(l, \varepsilon)\right)\left(l n-V_{o l}\right) \\
& =\sum_{j=1}^{k}\left(W_{j}+d(j, \varepsilon)\right)\left(j n-V_{o j}\right)=\sum_{j=1}^{k}\left(j n-V_{o j}\right) W_{j}+\sum_{j=1}^{k}\left(j n-V_{o j}\right) d(j, \varepsilon) .
\end{aligned}
$$


Therefore, Model (4) can be written as

$$
\begin{aligned}
\Theta_{o}^{*}=\max & \sum_{j=1}^{k} V_{o j} W_{j}+\sum_{j=1}^{k} V_{o j} d(j, \varepsilon), \\
\text { s.t. } \quad & \sum_{j=1}^{k} V_{o j} W_{j}+\sum_{j=1}^{k} V_{o j} d(j, \varepsilon) \leq 1, \\
& \sum_{j=1}^{k}\left(j n-V_{o j}\right) W_{j}+\sum_{j=1}^{k}\left(j n-V_{o j}\right) d(j, \varepsilon) \leq m-1, \\
& W_{j} \geq 0, \quad j=1, \ldots, k . \quad \square
\end{aligned}
$$

Proof of Theorem 1. Model (5) is equivalent to the following one:

$$
\begin{aligned}
\widetilde{\Theta}_{o}^{*}=\max & \sum_{j=1}^{k} V_{o j} W_{j}, \\
\text { s.t. } & \sum_{j=1}^{k} V_{o j} W_{j} \leq \delta_{o 1}, \\
& \sum_{j=1}^{k}\left(j n-V_{o j}\right) W_{j} \leq \delta_{o 2}, \\
& W_{j} \geq 0, \quad j=1, \ldots, k .
\end{aligned}
$$

Moreover, $\Theta_{o}^{*}=\widetilde{\Theta}_{o}^{*}+1-\delta_{o 1}$. It is well known that if a linear program has an optimal solution, then its dual also has an optimal solution and the optimal values for both problems are equal. Therefore, it is sufficient to solve the dual of the previous problem, that is,

$$
\begin{array}{ll}
\min & \delta_{o 1} X_{1}+\delta_{o 2} X_{2}, \\
\text { s.t. } & V_{o j} X_{1}+\left(j n-V_{o j}\right) X_{2} \geq V_{o j}, \quad j=1, \ldots, k, \\
& X_{1}, X_{2} \geq 0 .
\end{array}
$$

Let $S$ be the feasible set of this problem; i.e.,

$$
S=\left\{\left(X_{1}, X_{2}\right) \in \mathbb{R}_{+}^{2} \mid V_{o j} X_{1}+\left(j n-V_{o j}\right) X_{2} \geq V_{o j}, \quad j=1, \ldots, k\right\} .
$$

We distinguish two cases: 
1. If $V_{o 1}=n$, then $V_{o 2}=\cdots=V_{o k}=n$ and $S=\left\{\left(X_{1}, X_{2}\right) \in \mathbb{R}_{+}^{2} \mid X_{1} \geq 1\right\}$. Therefore $\widetilde{\Theta}_{o}^{*}=\delta_{o 1}$ and $\Theta_{o}^{*}=1$.

2. If $V_{o 1}<n$, then $V_{o j} X_{1}+\left(j n-V_{o j}\right) X_{2}=V_{o j}$ is the straight line that passes through the points $(1,0)$ and $\left(0, V_{o j} /\left(j n-V_{o j}\right)\right)$. Let $V_{o}^{*}$ and $p \in\{1, \ldots, k\}$ such that

$$
V_{o}^{*}=\max _{j=1, \ldots, k, k} \frac{V_{o j}}{j n-V_{o j}}=\frac{V_{o p}}{p n-V_{o p}} .
$$

Then $S=\left\{\left(X_{1}, X_{2}\right) \in \mathbb{R}_{+}^{2} \mid V_{o p} X_{1}+\left(p n-V_{o p}\right) X_{2} \geq V_{o p}\right\}$. We distinguish two cases:

(a) If $\delta_{o 2}=0$, then the objective function is $\delta_{o 1} X_{1}$ and an optimal solution is $\left(0, V_{o}^{*}\right)$. Therefore, $\widetilde{\Theta}_{o}^{*}=0$ and $\Theta_{o}^{*}=1-\delta_{o 1}$.

(b) If $\delta_{o 2} \neq 0$, then the contours of the objective function are straight lines with slope $-\delta_{o 1} / \delta_{o 2}$. We distinguish the following cases:

i. If $\delta_{o 1} / \delta_{o 2}<V_{o}^{*}$, then the optimal point is $(1,0)$ (see Figure A.2). Therefore $\widetilde{\Theta}_{o}^{*}=\delta_{o 1}$ and $\Theta_{o}^{*}=1$.

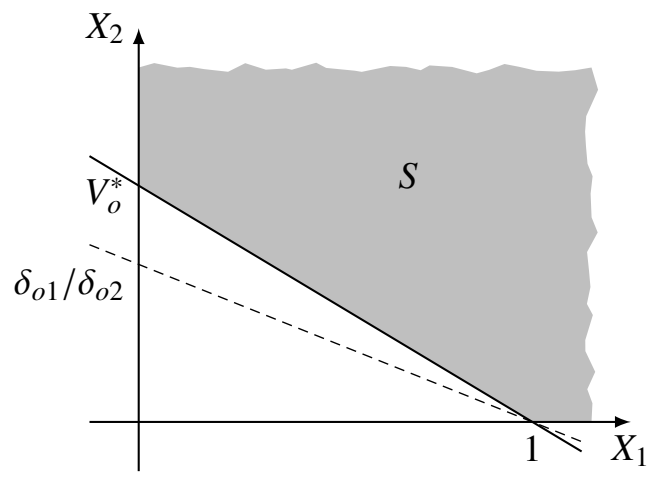

Figure A.2: Optimal point when $\delta_{o 1} / \delta_{o 2}<V_{o}^{*}$.

ii. If $\delta_{o 1} / \delta_{o 2}=V_{o}^{*}$, then the optimal solution is any point on the line segment between $(1,0)$ and $\left(0, V_{o}^{*}\right)$. In this case $\widetilde{\Theta}_{o}^{*}=\delta_{o 1}$ and $\Theta_{o}^{*}=1$.

iii. If $\delta_{o 1} / \delta_{o 2}>V_{o}^{*}$, then the optimal point is $\left(0, V_{o}^{*}\right)$. Therefore $\widetilde{\Theta}_{o}^{*}=\delta_{o 2} V_{o}^{*}$ and $\Theta_{o}^{*}=\delta_{o 2} V_{o}^{*}+$ $1-\delta_{o 1}$. 
Proof of Theorem 2. Model (7) is equivalent to the following one:

$$
\begin{aligned}
\widetilde{\Lambda}_{o}^{*}=\max & \sum_{j=1}^{k} V_{o j} W_{j}, \\
\text { s.t. } & \sum_{j=1}^{k}\left(j n-V_{o j}\right) W_{j} \leq \delta_{o 2}, \\
& W_{j} \geq 0, \quad j=1, \ldots, k .
\end{aligned}
$$

Just as in the previous proof, it suffices to solve the dual of this problem, that is,

$$
\begin{array}{ll}
\min & \delta_{o 2} X, \\
\text { s.t. } & \left(j n-V_{o j}\right) X \geq V_{o j}, \quad j=1, \ldots, k, \\
& X \geq 0 .
\end{array}
$$

It is easy to check that the optimal solution is $X^{*}=V_{o}^{*}=\max _{j=1, \ldots, k} \frac{V_{o j}}{j n-V_{o j}}$. Therefore, $\widetilde{\Lambda}_{o}^{*}=\delta_{o 2} V_{o}^{*}$ and $\Lambda_{o}^{*}=\delta_{o 2} V_{o}^{*}+\delta_{o}$.

Proof of Proposition 1. Given that $\max _{j=1, \ldots, k} \frac{V_{o j}}{j}=v_{o 1}>n / 2$ and $\max _{j=1, \ldots, k} \frac{V_{i j}}{j} \leq n / 2$ for all $i \neq o$, the proof is obvious from equivalence (9).

Proof of Proposition 2. If $d(j, \varepsilon)=g(j) \varepsilon$, then we have

$$
\begin{aligned}
\varepsilon_{o}^{*} & =\sup \left\{\varepsilon \geq 0 \mid \delta_{o 2}(\varepsilon) \geq 0\right\} \\
\varepsilon_{o}^{*} & =\sup \left\{\varepsilon \geq 0 \mid \sum_{j=1}^{k}\left(j n-V_{o j}\right) d(j, \varepsilon) \leq m-1\right\} \\
& =\sup \left\{\varepsilon \geq 0 \mid \varepsilon \sum_{j=1}^{k}\left(j n-V_{o j}\right) g(j) \leq m-1\right\} \\
& =\sup \left\{\varepsilon \geq 0 \mid \varepsilon \leq \frac{m-1}{\sum_{j=1}^{k}\left(j n-V_{o j}\right) g(j)}\right\} \\
& =\frac{m-1}{\sum_{j=1}^{k}\left(j n-V_{o j}\right) g(j)}
\end{aligned}
$$


and

$$
\begin{aligned}
& \widehat{\Lambda}_{o}=(m-1) V_{o}^{*}+\frac{1}{\varepsilon_{o}^{*}}\left(\sum_{j=1}^{k}\left(V_{o j}-\left(j n-V_{o j}\right) V_{o}^{*}\right) g(j)\right) \int_{0}^{\varepsilon_{o}^{*}} \varepsilon d \varepsilon \\
& =(m-1) V_{o}^{*}+\frac{\varepsilon_{o}^{*}}{2}\left(\sum_{j=1}^{k}\left(V_{o j}-\left(j n-V_{o j}\right) V_{o}^{*}\right) g(j)\right) \\
& =(m-1) V_{o}^{*}+\frac{m-1}{2 \sum_{j=1}^{k}\left(j n-V_{o j}\right) g(j)}\left(\sum_{j=1}^{k} V_{o j} g(j)-V_{o}^{*} \sum_{j=1}^{k}\left(j n-V_{o j}\right) g(j)\right) \\
& =(m-1) V_{o}^{*}+\frac{m-1}{2} \frac{\sum_{j=1}^{k} V_{o j} g(j)}{\sum_{j=1}^{k}\left(j n-V_{o j}\right) g(j)}-\frac{m-1}{2} V_{o}^{*} \\
& =\frac{m-1}{2}\left(V_{o}^{*}+\frac{\sum_{j=1}^{k} V_{o j} g(j)}{\left(n \sum_{j=1}^{k} j g(j)-\sum_{j=1}^{k} V_{o j} g(j)\right)}\right) \\
& =\frac{m-1}{2}\left(V_{o}^{*}+\left(\frac{n \sum_{j=1}^{k} j g(j)}{\sum_{j=1}^{k} V_{o j} g(j)}-1\right)^{-1}\right) \text {. }
\end{aligned}
$$

\section{References}

[1] Amin, G. R., \& Sadeghi, H. (2010). Application of prioritized aggregation operators in preference voting. Int. J. Intell. Syst., $25,1027-1034$.

[2] Andersen, P., \& Petersen, N. C. (1993). A procedure for ranking efficient units in data envelopment analysis. Management Sci., 39, 1261-1264.

[3] Brams, S. J., \& Fishburn, P. C. (2002). Voting procedures. In K. J. Arrow, A. K. Sen, \& K. Suzumura (Eds.), Handbook of Social Choice and Welfare chapter 4. (pp. 173-236). Elsevier volume 1 of Handbook of Social Choice and Welfare.

[4] Contreras, I. (2011). A DEA-inspired procedure for the aggregation of preferences. Expert Syst. Appl., 38, 564-570.

[5] Cook, W. D., \& Kress, M. (1990). A data envelopment model for aggregating preference rankings. Management Sci., 36, $1302-1310$

[6] Ebrahimnejad, A. (2012). A new approach for ranking of candidates in voting systems. OPSEARCH, 49, $103-115$. 
[7] Foroughi, A. A., \& Aouni, B. (2012). New approaches for determining a common set of weights for a voting system. Int. Trans. Oper. Res., 19, 521-530.

[8] Foroughi, A. A., Jones, D. F., \& Tamiz, M. (2005). A selection method for a preferential election. Appl. Math. Comput., 163, $107-116$.

[9] Foroughi, A. A., \& Tamiz, M. (2005). An effective total ranking model for a ranked voting system. Omega, 33, 491-496.

[10] Green, R. H., Doyle, J. R., \& Cook, W. D. (1996). Preference voting and project ranking using DEA and cross-evaluation. Eur. J. Oper. Res., 90, 461-472.

[11] Hashimoto, A. (1997). A ranked voting system using a DEA/AR exclusion model: A note. Eur. J. Oper. Res., 97, 600-604.

[12] Hashimoto, A., \& Wu, D.-A. (2004). A DEA-compromise programming model for comprehensive ranking. J. Oper. Res. Soc. Jpn., 47, 73-81.

[13] Hosseinzadeh Lotfi, F., \& Fallahnejad, R. (2011). A note on “A solution method to the problem proposed by Wang in voting systems". Appl. Math. Sci., 5, 3051-3055.

[14] Hosseinzadeh Lotfi, F., Rostamy-Malkhalifeh, M., Aghayi, N., Ghelej Beigi, Z., \& Gholami, K. (2013). An improved method for ranking alternatives in multiple criteria decision analysis. Appl. Math. Modell., 37, 25-33.

[15] Llamazares, B., \& Peña, T. (2009). Preference aggregation and DEA: An analysis of the methods proposed to discriminate efficient candidates. Eur. J. Oper. Res., 197, 714-721.

[16] Noguchi, H., Ogawa, M., \& Ishii, H. (2002). The appropriate total ranking method using DEA for multiple categorized purposes. J. Comput. Appl. Math., 146, 155-166.

[17] Obata, T., \& Ishii, H. (2003). A method for discriminating efficient candidates with ranked voting data. Eur. J. Oper. Res., $151,233-237$.

[18] Soltanifar, M., Ebrahimnejad, A., \& Farrokhi, M. M. (2010). Ranking of different ranking models using a voting model and its application in determining efficient candidates. Int. J. Soc. Syst. Sci., 2, 375-389.

[19] Stein, W. E., Mizzi, P. J., \& Pfaffenberger, R. C. (1994). A stochastic dominance analysis of ranked voting systems with scoring. Eur. J. Oper. Res., 74, 78-85.

[20] Wang, N. S., Yi, R. H., \& Liu, D. (2008). A solution method to the problem proposed by Wang in voting systems. J. Comput. Appl. Math., 221, 106-113.

[21] Wang, Y. M., \& Chin, K. S. (2007). Discriminating DEA efficient candidates by considering their least relative total scores. J. Comput. Appl. Math., 206, 209-215.

[22] Wang, Y. M., Chin, K. S., \& Yang, J. B. (2007). Three new models for preference voting and aggregation. J. Oper. Res. Soc., $58,1389-1393$.

[23] Wang, Y. M., Luo, Y., \& Hua, Z. (2007). Aggregating preference rankings using owa operator weights. Inform. Sci., 177, $3356-3363$.

[24] Wu, J., Liang, L., \& Zha, Y. (2009). Preference voting and ranking using DEA game cross efficiency model. J. Oper. Res. Soc. Jpn., 52, 105-111. 